\title{
The arrival of genomic medicine to the clinic is only the beginning of the journey
}

\author{
James P. Evans, MD, PhD ${ }^{1}$ and Muin J. Khoury, MD, $\mathrm{PhD}^{2}$
}

This month we are delighted to feature an important article titled "Implementing Genomic Medicine in the Clinic: The Future is Here." In this article, Teri Manolio and a veritable Who's Who of genomic medicine nicely articulate the nearterm promise of genomic medicine and lay out a number of current efforts being pursued at leading institutions, all designed to implement genomics in patient care. The authors detail many of the challenges to using genomics in clinical care and highlight four specific applications of genomics: tumorbased screening, family history-directed decision support, pharmacogenomics (PGx), and diagnostic genome sequencing to "demonstrate that genomic medicine is no longer on the threshold; it has arrived."

In doing so, the authors point toward many of the ways in which genomic medicine may ultimately benefit patients and the practice of medicine. They have not neglected to address many of the challenges and are not remiss in making multiple calls for an evidence-based approach to its implementation. Here, we wish to amplify these calls and emphasize the necessary primacy of evidence.

The speed with which genomics has become clinically relevant and the tremendous power of this new technology led to its rapid implementation in a variety of clinical settings. But as Manolio et al. ${ }^{1}$ remind us in their article, we must not assume that good ideas automatically translate into improved patient care. Their article should not be taken simply as a roadmap to genomic implementation for its own sake. Rather, it should serve as a roadmap for how to implement genomic medicine in a way that ultimately gets us to our real goal-a firm foundation of evidence-based genomic medicine.

In other words, the "arrival" of genomic medicine at its current stage represents less than half the journey. A few years ago, Khoury et al. ${ }^{2}$ identified four phases of genomics translational research starting with genome-based discoveries and leading to improved population health outcomes. In the context of genomic medicine, we have indeed arrived at the first destination (translational phase 1 (T1): from bench to bedside). In the T2 phase of translation, we must now figure out whether and how genomic analysis can actually improve patient outcomes and, specifically, which of the many promising facets of this new field offer real and tangible improvements in care. We should not prematurely skip this evaluation phase in favor of implementation research (T3) and outcomes research (T4). After all, from novel imaging modalities to new drugs, medicine is replete with tantalizing new approaches to patient care that promise marvels. Yet, the lessons of the history of medicine are clear: prematurely and casually implementing attractive technologies and strategies without meeting the hard and high bar of clearly improved outcomes is literally dangerous. The reflexive use of hormone replacement therapy ${ }^{3}$ in postmenopausal women and wholescale screening of men for prostate cancer by prostate-specific antigen measurement, ${ }^{4}$ to name just two recent examples, have arguably resulted in poor outcomes, needless anxiety, wasted effort, and untold cost-all at a time when the zero-sum landscape of health-care funding can least afford it.

A prominent application of genomics to patient care highlighted by Manolio et al. ${ }^{1}$, is the use of whole-genome sequencing to diagnose enigmatic conditions that have a high likelihood of a primary genetic etiology. This application is already becoming widespread; given that diagnosis is the lynchpin of clinical care, the bar that must be met for its implementation is low. It need only be shown that genomic approaches indeed yield answers, an outcome that in and of itself is of value to patients, clinicians, and researchers alike. What must now be defined are the specific clinical features of a patient's presentation that indicate that this technology is likely to yield a definitive diagnosis. In doing so, we will have a roadmap to guide clinicians as to whether it is likely to be worthwhile to bring genomics to bear on a given patient. This is no different from what must be done for any medical test. After all, magnetic resonance imaging is a marvelous and highly useful medical test. But we hear few (sensible) calls to obtain magnetic resonance imaging on all of our patients. Rather, research has defined those specific clinical settings in which a magnetic resonance imaging is likely to be productive. Therefore, genome-scale sequencing of patients will likely best be applied in specific and defined circumstances. Our field now faces the exciting challenge of defining those circumstances.

Other applications of genomics highlighted by Manolio et al. ${ }^{1}$ include the tantalizing prospects of whole-genome tumor sequencing to guide cancer therapeutics and the use of preemptive PGx testing to guide the selection of drugs. For both applications, evidence must now be sought that shows patient outcomes

${ }^{1}$ Department of Genetics, University of North Carolina at Chapel Hill, Chapel Hill, North Carolina, USA; ${ }^{2}$ Office of Public Health Genomics, Centers for Disease Control and

Prevention, Atlanta, Georgia, USA. Correspondence: James P. Evans (jpevans@med.unc.edu) 
improve before they are routinely employed; something that is hardly a foregone conclusion. On the tumor genomics side, we have had success stories with HER2 and EGFR to drive therapeutics for a subset of patients with cancer. Nevertheless, the sole example of germline PGx testing that has been recommended by an evidence-based panel $^{5}$ as standard of care (HLA-B ${ }^{\star 5701}$ testing before prescribing abacavir) is supported by a dramaticand virtually unique-odds ratio for association between an adverse event and a specific genotype, coupled with the existence of alternative treatment modalities. For other examples, even the most compelling scenarios in which PGx may improve outcomes remain largely speculative. Although many of us hope that CYP2C9 and VKORC1 genotyping may result in safer anticoagulation therapy or that clopidogrel can be more effectively used with PGx information in hand, no evidence-based groups have recommended their routine clinical use. Likewise, the use of whole-genome sequencing of tumors is an appealing application and could well prove to save lives and money. But it is not enough to hope; we must demonstrate that this is the case through sober, well-designed studies designed not to "show" that genomic medicine is wonderful but to simply and dispassionately investigate whether it really is. This is especially true in an era in which overtesting is increasingly being appreciated as a considerable threat to good outcomes and affordable care. ${ }^{6}$

We wish it were not so. It would be far cheaper and far easier if a high threshold for evidence with regard to outcomes was not needed. But the stakes are high. Premature implementation of even highly appealing medical modalities can carry a high price tag in terms of patient suffering and cost. In addition, although the cost of accruing such evidence is indeed high, it is trivial as compared with the cost of blind and uninformed implementation.

Part and parcel to the accrual of evidence is the development of new and innovative ways to evaluate evidence. As Manolio et al. ${ }^{1}$ articulate, we will never have enough time or money to conduct randomized controlled trials for each and every genotype in those myriad clinical settings in which genomics is likely to be relevant. We thus need to arrive at novel approaches for comparative effectiveness research, ${ }^{7}$ stakeholder engagement, ${ }^{8}$ and knowledge integration. ${ }^{9}$

Manolio et al. ${ }^{1}$ also address the thorny issue of cost, cogently arguing that new models are required to ensure adequate reimbursement for genomic analysis and implementation. Here too, evidence is the answer. When evidence of benefit is clearly demonstrated for a given approach, adequate reimbursement is far easier to secure. We must eschew "pushing" genomics into medicine because of our enthusiasm and hopes of its promise. Rather, we must focus on accruing evidence so that genomics-along with reimbursement-is "pulled" into patient care by demand from knowledgeable nongenetic physicians who clamor for its application to their patients because it has been shown to be a useful tool.

Critical to arriving at our ultimate destination-improved health outcomes as a result of implementation of genomic medicine-will be the construction of a genome-friendly electronic medical record. Manolio et al. ${ }^{1}$ rightly spend considerable time discussing the nature of the electronic medical record that will be required in a genomic age. It bears emphasizing that integrating genomic patient data into the electronic medical record should not be seen as an end in itself. Rather, it is a necessary first step that will then facilitate an evaluation of whether it can be of benefit to our patients.

The National Institutes of Health and especially the National Human Genome Research Institute have made an admirable start to facilitating the next phase of our genomic medicine journey. Through their funding of consortia such as those focused on clinical exploratory sequencing, medical genomic demonstration projects, and a new request for applications focused on defining clinically meaningful variation in the human genome, they are husbanding their resources wisely and maintaining a strong focus on evidence. In our appropriately celebratory mood regarding the "arrival" of genomic medicine, let's not forget that we still have a long way to go. The hard (and exciting) work of figuring out just how these wondrous new technologies can benefit our patients has only begun.

\section{REFERENCES}

1. Manolio TA, Chisolm R, Ozenberger B, et al. Implementing genomic medicine in the clinic: the future is here. Genet Med (in this issue).

2. Khoury MJ, Gwinn M, Yoon PW, Dowling N, Moore CA, Bradley L. The continuum of translation research in genomic medicine: how can we accelerate the appropriate integration of human genome discoveries into health care and disease prevention? Genet Med 2007;9:665-674.

3. U.S. Preventive Services Task Force. Hormone Replacement Therapy for the Prevention of Chronic Conditions in Postmenopausal Women. http:// www.uspreventiveservicestaskforce.org/uspstf/uspspmho.htm. Accessed 9 May 2012.

4. U.S. Preventive Services Task Force. Screening for Prostate Cancer: Current Recommendation. http://www.uspreventiveservicestaskforce.org/ prostatecancerscreening.htm. Accessed 9 May 2012.

5. Department of Health and Human Services Advisory Committee on HIV treatment 2012. http://aidsinfo.nih.gov/contentfiles/lvguidelines/ adultandadolescentgl.pdf. Accessed 7 September 2012.

6. Choosing Wisely. http://choosingwisely.org/. Accessed 9 May 2012.

7. Goddard KA, Knaus WA, Whitlock E, et al. Building the evidence base for decision making in cancer genomic medicine using comparative effectiveness research. Genet Med 2012; e-pub ahead of print 19 April 2012.

8. Deverka PA, Schully SD, Ishibe N, et al. Stakeholder assessment of the evidence for cancer genomic tests: insights from three case studies. Genet Med 2012; doi: 10.1038/gim.2012.3. e-pub ahead of print 5 April 2012.

9. Khoury MJ, Gwinn M, Dotson WD, Schully SD. Knowledge integration at the center of genomic medicine. Genet Med 2012; e-pub ahead of print 3 May 2012 